\title{
Surface plasmon resonance enhanced coupling to whispering-gallery modes in optical micropillar resonators
}

\author{
Nick K. Hon and Andrew W. Poon \\ Department of Electrical and Electronic Engineering, The Hong Kong University of Science and Technology \\ Clear Water Bay, Hong Kong SAR, China \\ Tel: (852)-2358-7905; Fax (852)-2358-1485; Email: eeawpoon@ust.hk
}

\begin{abstract}
We report surface plasmons enhanced coupling to whispering-gallery modes in micropillar resonators. We observe a 4 -fold coupling enhancement using a glass prism coated with a 20 -nm gold film and coupled to a silica micropillar resonator.

C2006 Optical Society of America

OCIS codes: (240.6680) Surface plasmons; (230.5750) Resonators
\end{abstract}

Recently, surface plasmon resonance (SPR) [1] enhanced optical phenomena have been attracting considerable interest in a wide range of applications including biochemical sensing $[2,3]$ and microscopy. Here we report initial experimental demonstrations of SPR enhanced coupling to whispering-gallery modes (WGMs) in silica micropillar resonators. Compared with microsphere resonators, micropillar resonators offer a long mm-cm length for coating with biochemical molecules for SPR-enhanced sensing [2].

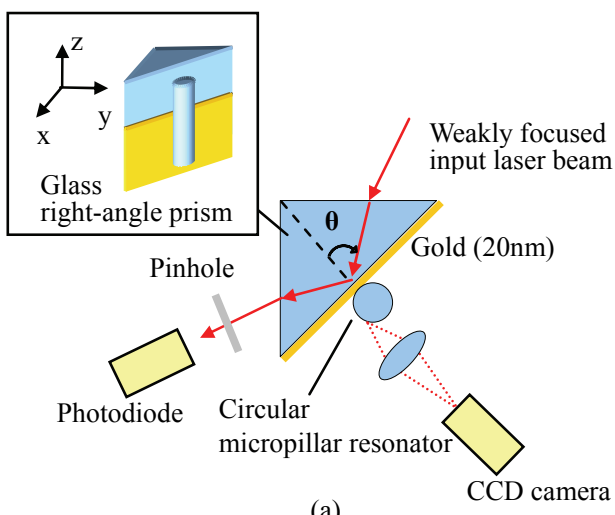

(a)

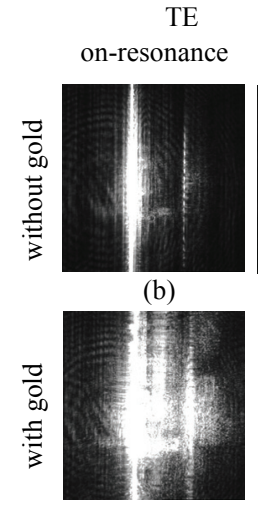

(d)

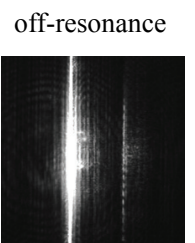

(c)

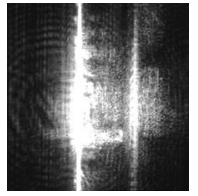

(e)
TM

on-resonance off-resonance

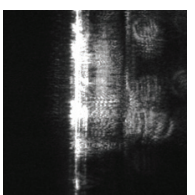

(f)

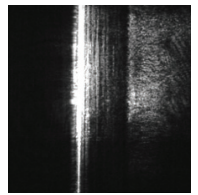

(h)

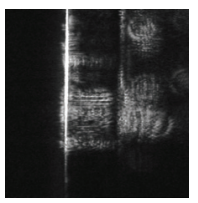

(g)

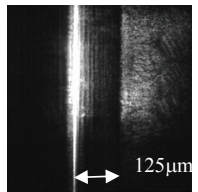

(i)

Fig. 1 (a) Schematic of the experimental setup using the conventional Kretschmann-Raether ATR coupling system. Inset illustrates a right-angle prism with the lower half of the prism surface sputtered with a 20-nm gold thin film. (b) - (i) show typical images of the circular micropillar resonator taken on the transmission side at $\theta \sim 49.18^{\circ}$.

Fig. 1(a) shows the schematic of our experimental setup using the conventional Kretschmann-Raether ATR coupling system. We use a glass (BK7) right-angle prism with a $20-\mathrm{nm}$ thin gold film sputtered on the prism surface. We employ a standard singlemode silica optical fiber as a $125-\mu \mathrm{m}$-diameter micropillar resonator. The micropillar resonator is attached onto the gold-coated prism surface. Only the lower half of the prism is sputtered with gold (inset) in order to enable control experiments without gold-film coating on the same prism-fiber setup. A laser beam from an external-cavity wavelength tunable diode laser $(675 \mathrm{~nm}-693 \mathrm{~nm})$ is weakly focused onto the prism-fiber coupling surface at an incident angle $\theta$ above the critical angle for total internal reflection. We use TE-polarization to excite SPR effects. We measure the internal reflection spectra at the far field after a 50- $\mu$-size pinhole using a photodiode. We also image the fiber sidewall transmission onto a CCD camera.

Figs. 1(b) - 1(e) show typical fiber images taken at the transmission side in TE polarization. We compare the transmission intensity levels between typical on- and off-resonance wavelengths in the same z region (both with and without gold coating). We see that the on-resonance image with gold coating (Fig. 1(d)) exhibits a pronounced enhancement compared with the off-resonance image (Fig. 1(e)). Whereas, the on-resonance image without gold coating (Fig. 1(b)) only exhibits a marginal enhancement compared with the off-resonance image (Fig. 1(c)). We also image the fiber transmission in TM polarization (by rotating the laser beam polarization from TE to TM), as shown in Figs. 1(f) - 1(i). While the on-resonance intensity (Fig. 1(f)) is enhanced compared with the 
off-resonance intensity (Fig. 1(g)) in the z-region without gold coating, the on-resonance intensity (Fig. 1(h)) is essentially the same as the off-resonance intensity (Fig. 1(i)) in the z-region with gold coating.

Fig. 2(a) shows two measured TE-polarized reflection spectra (in $\log$ scale) at $\theta \approx 48.5^{\circ}$. The multimode spectrum (red) from a z-position with gold coating exhibits more pronounced resonance dips than the control multimode spectrum (blue). Mode A with gold coating displays a coupling efficiency of $\sim 70 \%$, whereas mode B (control) at essentially the same wavelength only reveals a coupling efficiency of $\sim 22 \%$. Both modes A and B have similarly high $\mathrm{Q} \sim 10^{4}$.

Fig. 2(b) shows the measured coupling enhancement factor (normalized coupling efficiency between modes A and $B$ ) as a function of $\theta$. We observe a maximum enhancement factor of 4 near $\theta=48^{\circ}$ with an angular width of $\sim 4.3^{\circ}$. We confirm that the coupling enhancement is due to SPR by comparing with the normalized reflected light intensity (reflectance) at an off-resonance wavelength $(\sim 687 \mathrm{~nm})$ as a function of $\theta$, as shown in the inset of Fig. 2(b). We observe a pronounced reflectance dip at $\theta \approx 47.8^{\circ}$ with an angular full-width half-maximum of $\sim 5.4^{\circ}$, which is consistent with our observed angular span of the enhanced WGM coupling. We remark that TM-polarized internal reflection spectra from gold-coated prism-fiber coupling only display attenuated resonances.
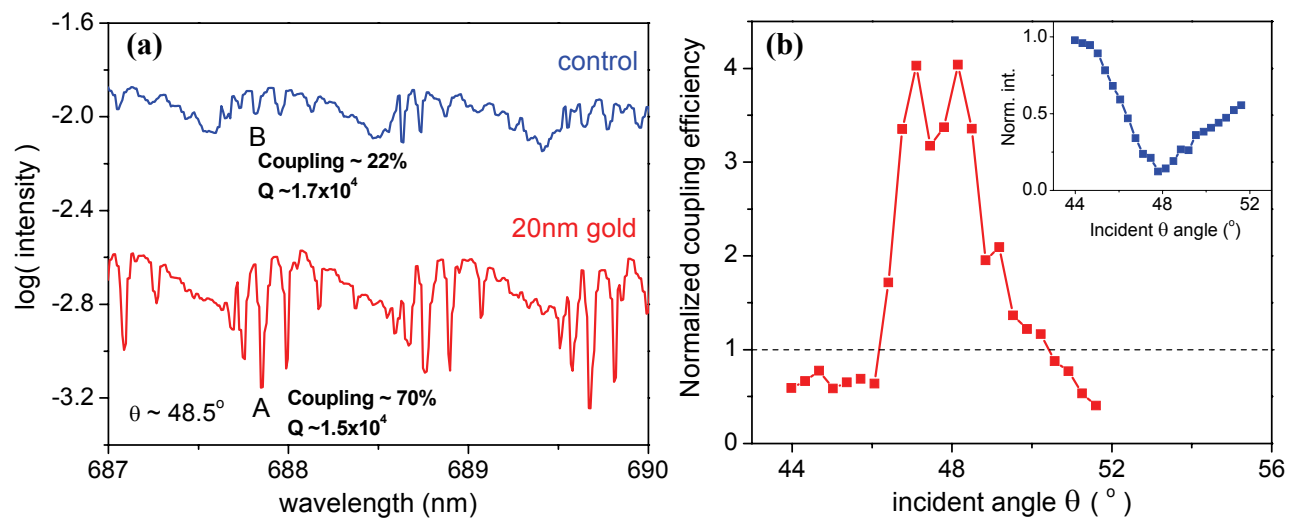

Fig. 2 (a) Measured TE-polarized reflection spectra at $\theta \sim 48.5^{\circ}$. The red curve is taken from a region with 20-nm gold coating. The blue curve is taken from a region without gold-film coating. (b) The normalized coupling efficiency of mode A to mode B at various incident angles. Inset shows the measured surface plasmon resonance reflectance dip.

In summary, we demonstrated surface plasmon resonance enhanced coupling to whispering-gallery modes in a large-sized silica optical micropillar resonator. We observed a maximum of 4-fold coupling enhancement in TE polarization by using a 20 -nm-thin gold film coated glass prism that is coupled to a 125 - $\mu \mathrm{m}$-diameter micropillar resonator. We observe that the angular span of the coupling enhancement is consistent with the measured surface plasmon resonance reflectance dip. Our on-going experiments focus on optimizing the gold-film thickness and also exploring plasmon enhanced coupling to polygonal micropillar resonators. Future experiments will investigate sensitizing the silica glass fiber for surface plasmon resonance enhanced WGM-based bio-molecules sensing.

\section{References}

[1] H. Raether, Surface Plasmons on Smooth and Rough Surfaces and on Gratings (Springer-Verlag, Berlin, 1988).

[2] D. Amarie, T.-D. Onuta, R. A. Potyrailo, and B. Dragnea, “Submicrometer Cavity Surface Plasmon Sensors," J. Phys. Chem. B 109, 15515-15519 (2005).

[3] K. Choi, H. Kim, Y. Lim, S. Kim, and B. Lee, "Analytic design and visualization of multiple surface plasmon resonance excitation using angular spectrum decomposition for a Gaussian input beam," Opt. Express 13, 8867 (2005). 